\title{
Rosai-Dorfman disease presenting as a solitary soft-tissue mass in the thigh: a case report
}

\author{
Alice KY Au1 *, FHKCR, FHKAM (Radiology), HM Cheng ${ }^{1}$, FHKCR, FHKAM (Radiology), KY Cho', FHKCR, FHKAM (Radiology), \\ CW Tam¹, FHKCR, FHKAM (Radiology), Jennifer LS Khoo', FHKCR, FHKAM (Radiology), Joshua HY Ng², MB, BS, \\ Vincent TW Hau ${ }^{3}$, MB, ChB, FHKAM (Orthopaedic Surgery)
}

${ }^{1}$ Department of Radiology, ${ }^{2}$ Department of Clinical Pathology, ${ }^{3}$ Department of Orthopaedics and Traumatology, Pamela Youde Nethersole Eastern Hospital, Chai Wan, Hong Kong

Hong Kong Med J 2019;25:149-51

*Corresponding author: augar520@gmail.com

https://doi.org/10.12809/hkmj164921

\section{Case report}

A 46-year-old man presented to the Department of Orthopaedics and Traumatology, Pamela Youde Nethersole Eastern Hospital in February 2004 with a 3-month history of self-detected left thigh mass. It was of spontaneous onset with no history of trauma, associated pain, weakness, or numbness. The patient had full range of movement and no lymphadenopathy was noted. Magnetic resonance imaging (MRI) [Fig 1] revealed a large area of infiltrative soft-tissue
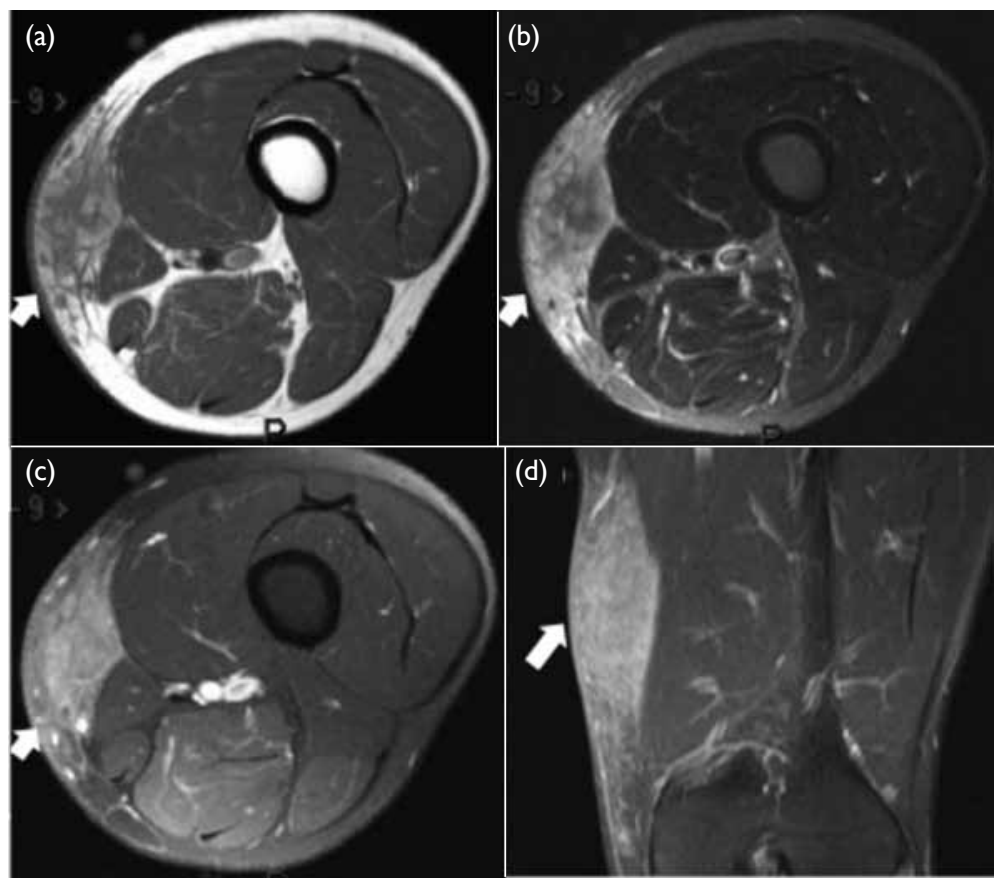

FIG I. (a) Axial TI-weighted magnetic resonance (MR) image showing a large area of infiltrative soft tissue thickening involving the subcutaneous layer at the medial aspect of the thigh with hypo- to iso-intense signals (arrow). (b) Axial T2-weighted fat-suppressed MR image showing hyperintense signals with internal heterogeneity and foci of hypointensities. Internal reticular pattern of septal thickening was present (arrow). (c, d) Axial and coronal TI-weighted fat-suppressed post-contrast MR images showing gadolinium enhancement of the lesion (arrow). The margin of the lesion was well delineated from the underlying vastus medialis and sartorius muscles without features of muscular invasion or destruction (arrow) thickening at the medial aspect of the left distal thigh and involved the subcutaneous layer. The lesion measured $8.4 \times 3.4 \times 11.2 \mathrm{~cm}$ (anteroposterior $\times$ transverse $\times$ longitudinal) and was characterised by T1-weighted (T1W) hypointense to isointense and T2-weighted (T2W) fat-suppressed hyperintense signals with internal heterogeneity. Internal foci of hypointensity in the T2W fat-suppressed images were noted. An internal reticular pattern of septal thickening was also found. There was enhancement after gadolinium contrast administration. The margin of the lesion was well delineated from the underlying vastus medialis and sartorius muscles with no features of muscular invasion or destruction. The knee joint was unremarkable and bone marrow signal was normal. The neurovascular bundle was also intact. Overall features were non-specific for either inflammatory or neoplastic pathology.

Microscopic examination of an incisional biopsy over the left vastus medialis with a wedge of skin and subcutaneous tissue revealed infiltrate in the subcutis and to a lesser extent the deep dermis. The infiltrate consisted of lymphocytes and a low number of plasma cells. Immunohistochemical stains showed mainly T-cells and some B-cells. Occasional areas with aggregates of paler histiocytic cells were present and suggested granuloma formation. Stains for acid-fast bacilli and fungus were negative. The paler histiocytic cells were S100-positive and showed lymphophagocytosis (Fig 2). Molecular study by polymerase chain reaction showed no clonal T-cell proliferation. The overall features were suggestive of Rosai-Dorfman disease (RDD).

Radical excision of the lesion was performed subsequently and included the epimysium of the gracilis, sartorius and aponeurosis of the vastus medialis. The excision margin in the radial excision of the lesion was $2 \mathrm{~cm}$. Microscopic examination revealed that the mass in the subcutis was composed of nodules or aggregates of lymphohistiocytic cells separated by areas of fibrosis. The cellular aggregates were composed of dark areas with plasma cells and lymphocytes and pale areas with 

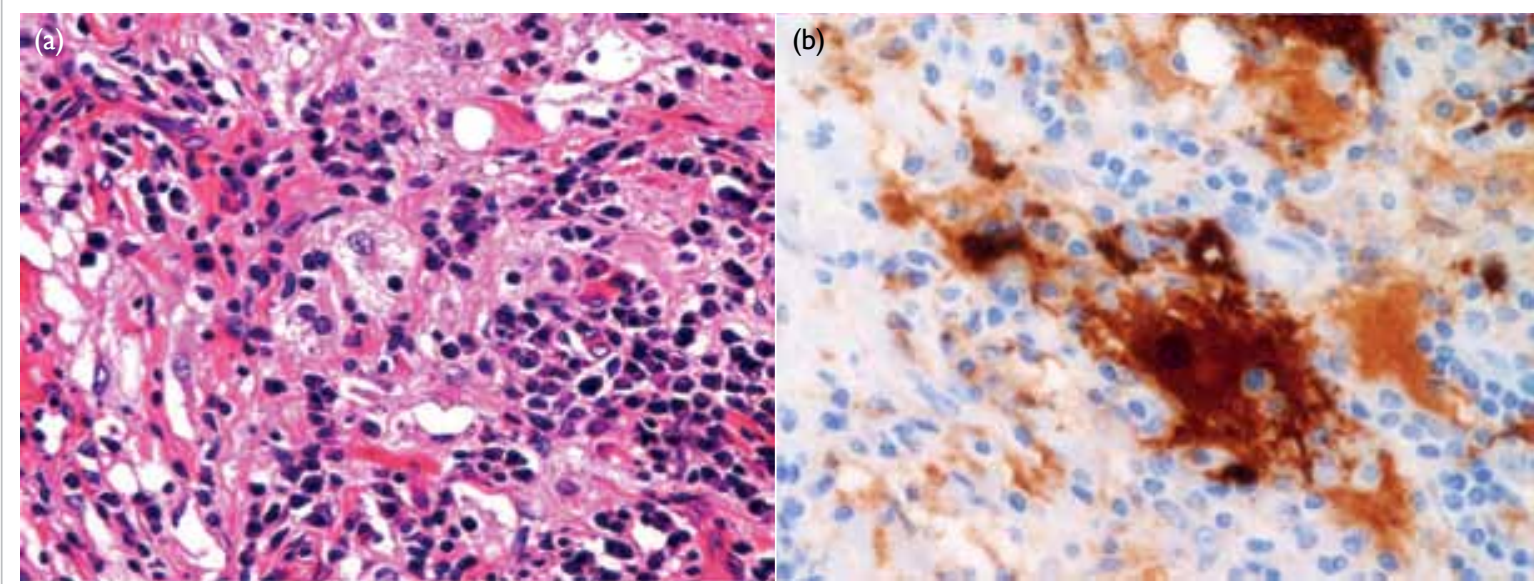

FIG 2. (a) Pale areas of histiocytes with emperipolesis and adjacent areas rich in lymphocytes and plasma cells are evident (haematoxylin and eosin, $\times 400$ ). (b) Histiocytes are S100-positive on immunostaining. Emperipolesis can also be demonstrated (immunostain for $\mathrm{SI00} \times \mathbf{6 0 0}$ )
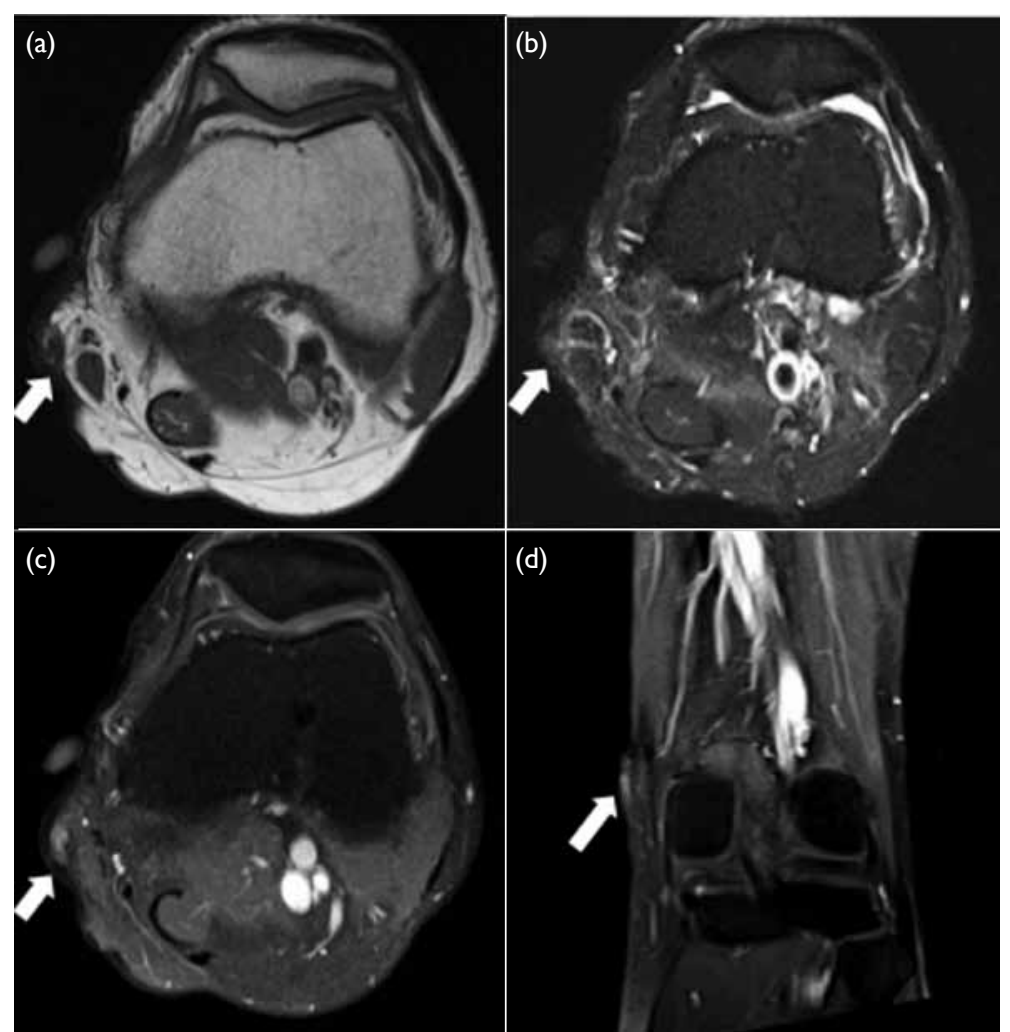

FIG 3. (a) Axial TI-weighted (TIW) magnetic resonance (MR) image showing an 8-mm nodular hypointense lesion at the inferior margin of the operative site. (b) Axial T2-weighted fat-suppressed MR image showing an isointense to mildly hyperintense lesion (arrow). (c, d) Axial and coronal TI-weighted fat-suppressed post-contrast MR images showing nodular enhancement of the lesion (arrow). Features could represent postoperative change or tumour recurrence (arrow) (phagocytosis of plasma cells and lymphocytes). The histiocytes showed positive immunostaining for S100. Special stains for acid-fast bacilli and fungus were again negative. The overall features were consistent with RDD. The resection margins were unremarkable. The patient recovered well postoperatively.

Eight years after the operation, the patient detected a nodular swelling over the inferior margin of the surgical site. Serial MRI showed a static nodular T1W hypointense, and T2W isointense to mildly hyperintense soft-tissue lesion with contrast enhancement, measuring approximately $8 \mathrm{~mm}$ in diameter (Fig 3). Features could represent postoperative change or tumour recurrence. The patient was otherwise asymptomatic and he opted for follow-up scans to monitor the lesion instead of surgical excision.

\section{Discussion}

Rosai-Dorfman disease is also known as sinus histiocytosis with massive lymphadenopathy and was first described by Rosai and Dorfman in $1969 .{ }^{1}$ It is a rare non-malignant histiocytic proliferative disorder. Although the disease may develop at any age, it is more common in young adults with a mean age of onset of 20 years and a slight male predominance $(1.4: 1) .{ }^{2,3}$

The aetiology of RDD is unknown, although previous studies have attempted to relate RDD to infectious agents including Epstein-Barr virus, human herpesvirus 6, herpes simplex virus, Brucella, Klebsiella rhinoscleromatis, and Nocardia. ${ }^{4}$ The disease involves a wide distribution and can affect a multitude of organ systems, including nodal involvement and extranodal involvement. The 
majority of patients present with painless massive cervical lymphadenopathy. Most patients have a complete and spontaneous remission, but some may experience recurrent or persistent albeit stable lymphadenopathy. In rare cases, the disease may follow an aggressive course and be fatal.

Pure cutaneous RDD is a distinct clinical entity that has an older age of onset (median 43.5 years) and a male-to-female ratio of $1: 2 .^{3}$ In contrast to systemic RDD that is commonly seen in blacks and rarely reported in Asians, most patients with purely cutaneous RDD are Asians or whites. The lesion remains localised to the skin even after long-term follow-up. ${ }^{5}$

Histologically, RDD is characterised by sheets of large pale histiocytes with large, round, vesicular nuclei. Phagocytosis of lymphoid cells or neutrophils by histiocytes may be found ("emperipolesis"). Immunohistochemical stains are useful when diagnosing RDD and the most consistent and reliable phenotype for $\mathrm{RDD}$ is $\mathrm{S} 100$ positive and CD1a negative.

Relative to the wide disease spectrum, there are variable radiographic features. Although no specific imaging characteristics allow differentiation of lymphadenopathy in RDD from the myriad other disease processes, massive painless bilateral cervical lymph node enlargement, particularly when it occurs in children and adolescents, should prompt consideration of RDD as a differential diagnosis. Nodal involvement may be evidenced as lymphadenopathy. In computed tomography scan of the sinuses and brain, polypoid masses, mucosal thickening, soft-tissue lesion of the paranasal sinuses or nasal cavity with or without associated osseous erosion can be seen. Features of brain involvement include a hyperattenuating meningeal-based mass showing contrast enhancement or parenchymal oedema surrounding the lesion. In MRI of the sinuses and brain, sinus lesions may also demonstrate hypointensity on T2W images. Meningeal-based mass lesions may demonstrate $\mathrm{T} 1 \mathrm{~W}$ isointensity to grey matter, $\mathrm{T} 2 \mathrm{~W}$ hyperintensity to grey matter and homogeneous contrast enhancement. ${ }^{6}$ Gallium scanning may show increased uptake and increased metabolism with fluorodeoxyglucose positron emission tomography. The differential diagnosis is broad and includes infectious (granulomatous) disease, Wegener's granulomatosis, other histiocytosis, Hodgkin's and non-Hodgkin's lymphoma, and fibroinflammatory lesions. In general, RDD does not show bone or soft-tissue destruction as in cases of Wegener's granulomatosis and T-cell lymphoma.

Rosai-Dorfman disease usually follows a benign and self-limiting course with treatment largely targeted at controlling local manifestations. Surgical options may be warranted for symptomatic control.

\section{Author contributions}

All authors contributed to the concept or design, acquisition of data, analysis or interpretation of data, drafting of the manuscript, and critical revision for important intellectual content. All authors had full access to the data, contributed to the study, approved the final version for publication, and take responsibility for its accuracy and integrity.

\section{Conflicts of interest}

All authors have disclosed no conflicts of interest.

\section{Funding/support}

This research received no specific grant from any funding agency in the public, commercial, or not-for-profit sectors.

\section{Ethics approval}

This study was conducted in accordance with the Declaration of Helsinki. The patient provided written informed consent.

\section{References}

1. Rosai J, Dorfman RF. Sinus histiocytosis with massive lymphadenopathy. A newly recognized benign clinical pathologic entity. Arch Pathol 1969;87:63-70.

2. Annessi G, Giannetti A. Purely cutaneous Rosai-Dorfman disease. Br J Dermatol 1996;134:749-53.

3. Brenn T, Calonje E, Granter SR, et al. Cutaneous RosaiDorfman disease is a distinct clinical entity. Am J Dermatopathol 2002;24:385-91.

4. Lu CI, Kuo TT, Wong WR, Hong HS. Clinical and histopathologic spectrum of cutaneous Rosai-Dorfman disease in Taiwan. J Am Acad Dermatol 2004;51:931-9.

5. Farooq U, Chacon A, Vincek V, Elgart GW. Purely cutaneous Rosai-Dorfman disease with immunohistochemistry. Indian J Dermatol 2013;58:447-50.

6. Symss NP, Cugati G, Vasudevan MC, Ramamurthi R, Pande A. Intracranial Rosai Dorfman disease: report of three cases and literature review. Asian J Neurosurg 2010;5:1930 . 